\title{
Sulfidative Purification of Carbon Nanotubes Integrated in Transistors
}

Yo-Sep Min, Eun Ju Bae, and Wanjun Park*

Materials \& Devices Research Center, Samsung Advanced Institute of Technology, Yong-In, Kyeongki-Do, 449-712, Korea

E-mail: wanjun@samsung.com

\section{Supporting Information}

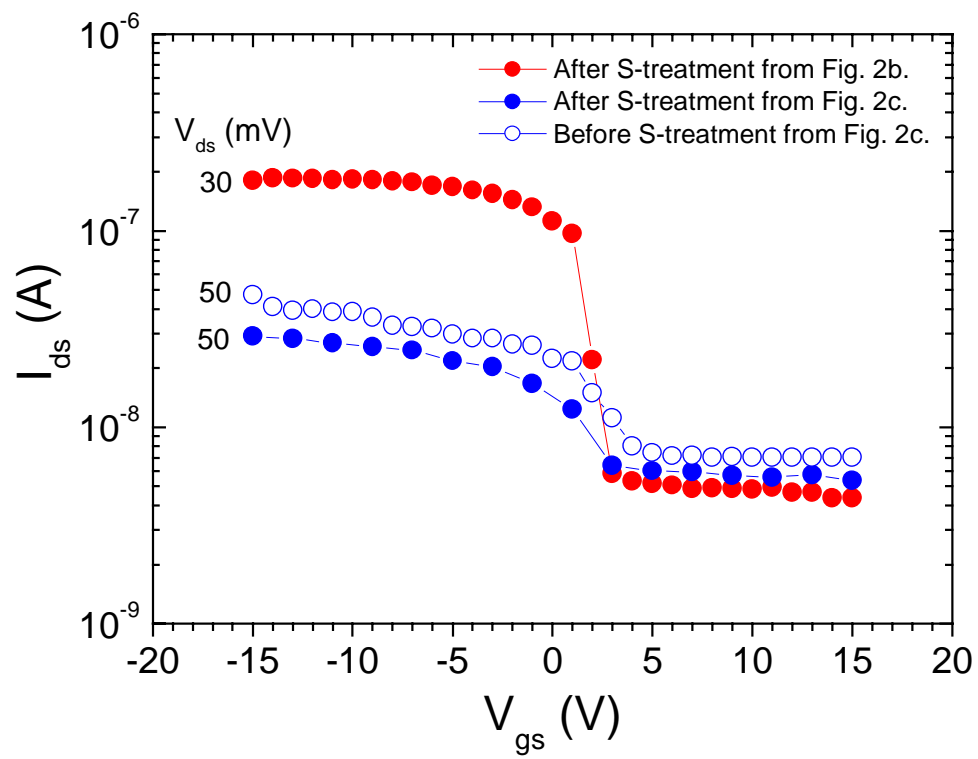

Figure S1. $\mathrm{I}_{\mathrm{ds}}-\mathrm{V}_{\mathrm{gs}}$ curves in a log scale before (open circles) and after (solid circles) sulfur treatment. Red and blue curves are re-plotted from Figure $2 b$ and $2 c$, respectively. The transistor with high offstate current before the sulfur treatment turns on and off at $3 \mathrm{~V}$ after treatment as shown with red circles in which the on-state and off-state currents are on the order of $\sim 10^{-7} \mathrm{~A}$ and $\sim 10^{-9} \mathrm{~A}$, respectively. The normally operating transistor before the sulfur treatment shows nearly the same on-/off-state current ratio after the treatment (blue circles). Note that the small on/off ratios are attributed to the large diameter $(\sim 2 \mathrm{~nm})$ of the nanotube integrated in the transistor [Ref S1]. 


\section{CNT-FET Fabrication Procedure}

Single-crystal Si substrate ( $p$-type), with a resistivity of $0.005 \sim 0.01 \Omega \mathrm{cm}$, were cleaned and coated with $300 \mathrm{~nm}$ of thermal $\mathrm{SiO}_{2}$. Nanotubes were dispersed from a 1,2-dichloroethane solution by spinning onto the substrates after mild sonication. The concentration of the solution was adjusted to yield approximately one nanotube in an area of $5 \times 5 \mu \mathrm{m}^{2}$. The spin-coating was performed with a rotation speed of $2000 \mathrm{rpm}$ for $30 \mathrm{sec}$ and subsequently $4000 \mathrm{rpm}$ for $10 \mathrm{sec}$. Alignment marks consisting of a metal dot array with $\sim 1 \mu \mathrm{m}$ spacing were fabricated to identify the location of a nanotube. A suitable nanotube was selected by using a scanning probe microcope. Once a suitable nanotube was found, its position was determined relative to the alignment marks. After generating patterns for metal contacts by electron-beam lithography, Pd electrodes of $100 \mathrm{~nm}$ were defined by liftoff process.

\section{Reference}

S1. Rakitin, A.; Papadopoulos, C.; Xu, J.M. Phys. Rev. B 2000, 61, 5793. 